\title{
THE STABILITY OF KY FAN'S POINTS
}

\author{
KOK-KEONG TAN, JIAN YU, AND XIAN-ZHI YUAN
}

(Communicated by Palle E. T. Jorgensen)

\begin{abstract}
In this paper, inspired by the work of Fort, the stability of the set $F(f)=\left\{y \in X: \sup _{x \in X} f(x, y) \leq 0\right\} \quad$ (respectively, the set $F(A, f)=\{y \in$ $\left.A: \sup _{x \in A} f(x, y) \leq 0\right\}$ ) with $f$ varying (respectively, with both $f$ and $A$ varying) is studied where $X$ is a non-empty compact convex subset of a Hausdorff topological vector space (respectively, $X$ is a Hausdorff topological space and $A$ is a non-empty compact subset of $X$ ) and $f: X \times X \rightarrow \mathbb{R}$ is bounded.
\end{abstract}

\section{INTRODUCTION}

Let $(X, d)$ be a compact metric space with fixed point property for continuous mappings. In [6], Fort introduced the concept of essential fixed points of a continuous mapping $f$ on $X$. He proved that (1) every continuous mapping on $X$ can be arbitrarily approximated by a continuous mapping on $X$ whose fixed points are all essential and (2) if each fixed point of a continuous mapping $f$ on $X$ is essential, then the fixed point set $F(f)=\{x \in X: f(x)=x\}$ of $f$ is stable: for each $\epsilon>0$, there is $\delta>0$ such that for each continuous mapping $g$ on $X$, if $\rho(f, g)=\sup \{d(f(x), g(x)): x \in X\}<\delta$, then $h(F(f), F(g))<\epsilon$ where $h$ is the Hausdorff metric defined on all bounded closed subsets of $X$ induced by the metric $d$; i.e., the fixed point set $F(g)$ of $g$ is "close" to the fixed point set $F(f)$ of $f$ whenever $g$ is "close" to $f$.

On the other hand, in [4], Ky Fan proved the following minimax inequality which plays a very important role in nonlinear analysis (e.g., see Lin and Simons [9]):

Theorem A. Let $X$ be a non-empty compact convex subset of a Hausdorff topological vector space and $f: X \times X \rightarrow \mathbb{R}$ be such that

(a) for each fixed $x \in X, y \mapsto f(x, y)$ is lower semicontinuous;

(b) for each fixed $y \in X, x \mapsto f(x, y)$ is quasi-concave (i.e., for each $\lambda \in \mathbb{R}$, the set $\{x \in X: f(x, y)>\lambda\}$ is convex).

Then $\inf _{y \in X} \sup _{x \in X} f(x, y) \leq \sup _{x \in X} f(x, x)$.

Received by the editors July 26, 1993 and, in revised form, August 27, 1993.

1991 Mathematics Subject Classification. Primary 46A55, 47H04, 47H10, 49J40, 52A05, 52A07.

Key words and phrases. Vietoris topology, $K F$ points, Cech-complete, stability, essential, minimax inequality, almost lower semicontinuous. 
It is clear that Ky Fan's minimax inequality Theorem $\mathrm{A}$ is equivalent to the following:

Theorem B. Let $X$ be a non-empty compact convex subset of a Hausdorff topological vector space and $f: X \times X \rightarrow \mathbb{R}$ be such that

(i) $f(x, x) \leq 0$ for all $x \in X$;

(ii) for each fuxed $x \in X, y \mapsto f(x, y)$ is lower semicontinuous;

(iii) for each fixed $y \in X, x \mapsto f(x, y)$ is quasi-concave.

Then there exists $\hat{y} \in X$ such that $f(x, \hat{y}) \leq 0$ for all $x \in X$.

We shall call such a point $\hat{y}$ in Theorem B the Ky Fan's point (in short, $K F$ point) of $f$ in $X$ and denote by $F(f)$ the set of all $K F$ points of $f$ in $X$. Thus $F(f)$ is non-empty by Theorem B. Also, by the condition (ii) of Theorem B, $F(f)=\cap_{x \in X}\{y \in X: f(x, y) \leq 0\}$ is closed in $X$ and is thus also compact. Therefore, for each function $f: X \times X \rightarrow \mathbb{R}$ satisfying the conditions (i), (ii) and (iii) of Theorem B, one can associate a non-empty compact subset $F(f)$, the set of all solutions $y \in X$ of the inequality $\sup _{x \in X} f(x, y) \leq 0$, of $X$.

In this paper, we shall first discuss the stability of $F(f)$ with $f$ varying where $f$ is a bounded real-valued function on $X \times X$ satisfying the conditions (i), (ii), and (iii) in Theorem B and $X$ is a non-empty compact convex subset of a Hausdorff topological vector space. Next, if $X$ is a Cech-complete space which belongs to the class $\mathscr{L}$ (see definition below), we shall study the stability of the set $F(A, f)=\left\{y \in A: \sup _{x \in A} f(x, y) \leq 0\right\}$ with both $f$ and $A$ varying, where $f: X \times X \rightarrow \mathbb{R}$ is bounded and lower semicontinuous and $A$ is a nonempty compact subset of $X$. When $X$ is a closed convex subset of a Frechet space, as an application, the stability of the set $F(A, f)$ is investigated, where $f: X \times X \rightarrow \mathbb{R}$ satisfies, in addition, the conditions (i) and (iii) of Theorem $\mathrm{B}$ and the subset $A$ is, in addition, compact convex.

Now we shall recall some definitions. If $X$ is a topological space, we shall denote by $K(X)$ and $\mathscr{P}_{0}(X)$ the space of all non-empty compact subsets of $X$ and the space of all non-empty subsets of $X$ respectively, both endowed with the Vietoris topology (see Klein and Thompson [8]). If $Z$ is another topological space, then a mapping $T: X \rightarrow \mathscr{P}_{0}(Z)$ is said to be (i) upper (respectively, lower) semicontinuous at $x \in X$, if for each open set $G$ in $Z$ with $G \supset T(x)$ (respectively, $G \cap T(x) \neq \varnothing$ ), there exists an open neighborhood $O(x)$ of $x$ in $X$ such that $G \supset T\left(x^{\prime}\right)$ (respectively, $G \cap T\left(x^{\prime}\right) \neq \varnothing$ ) for each $x^{\prime} \in O(x)$; (ii) $T$ is said to be almost lower semicontinuous at $x \in X$, if there exists $z \in T(x)$ such that for each open neighborhood $N(z)$ of $z$ in $Z$, there exists an open neighborhood $O(x)$ of $x$ in $X$ with the property that $N(z) \cap T\left(x^{\prime}\right) \neq \varnothing$ for each $x^{\prime} \in O(x)$; and (iii) $T$ is an usco if $T$ is upper semicontinuous with non-empty compact values. Also, the space $X$ is said (i) to be Cech-complete if it can be embedded as a $G_{\delta}$ subset of some compact Hausdorff space and (ii) to belong to the class $\mathscr{L}$ (see Kenderov [7]) if for each Cech-complete space $Z$, every usco mapping $F: Z \rightarrow K(X)$ is almost lower semicontinuous on some dense $G_{\delta}$ subset of $Z$.

By Theorem 2 of Fort [5], each completely metrizable space belongs to $\mathscr{L}$ and each Banach space with its weak topology (which is non-metrizable if it is infinite dimensional) is also in $\mathscr{L}$ (see Theorem 2 of Christensen [2]). 


\section{Stability IN THE COMPACT SETting}

Throughout this section, $X$ denotes a non-empty compact convex subset of a Hausdorff topological vector space. Let $L(X)$ be the family of all bounded real-valued functions on $X \times X$. For $f, g \in L(X)$, define

$$
\rho(f, g)=\sup _{x, y \in X}|f(x, y)-g(x, y)| \text {. }
$$

Clearly, $(L(X), \rho)$ is a complete metric space. Let

$$
M=\{f \in L(X): f \text { satisfies the conditions (i), (ii), and (iii) of Theorem B }\} \text {. }
$$

It is easy to show that $M$ is closed in $L(X)$. Thus we have:

Lemma 2.1. $(M, \rho)$ is a complete metric space.

Now for each $f \in M$, the set $F(f)=\left\{y \in X: \sup _{x \in X} f(x, y) \leq 0\right\}$ is non-empty and compact by Theorem B. Furthermore, we have:

Lemma 2.2. $F: M \rightarrow K(X)$ is upper semicontinuous.

Proof. Since $X$ is compact, we only need to prove that the graph of $F$ is closed in $M \times X$. For any net $\left\{\left(f_{\alpha}, y_{\alpha}\right)\right\}_{\alpha \in \Gamma}$ in Graph $F$ with $\left(f_{\alpha}, y_{\alpha}\right) \rightarrow$ $\left(f, y_{0}\right) \in M \times X$, we have $f_{\alpha} \rightarrow f, y_{\alpha} \rightarrow y_{0}$ and $f_{\alpha}\left(x, y_{\alpha}\right) \leq 0$ for all $\alpha \in \Gamma$ and for all $x \in X$. Fix $x \in X$. Since $y \mapsto f(x, y)$ is lower semicontinuous at $y_{0}$, for any $\epsilon>0$, there exists an open neighborhood $O\left(y_{0}\right)$ of $y_{0}$ in $X$ such that for each $y^{\prime} \in O\left(y_{0}\right), f\left(x, y_{0}\right)<f\left(x, y^{\prime}\right)+\epsilon / 4$. As $f_{\alpha} \rightarrow f$, there exists $\alpha_{0} \in \Gamma$ such that for any $\alpha \geq \alpha_{0}, \rho\left(f, f_{\alpha}\right)<\epsilon / 4$ so that for each $y^{\prime} \in O\left(y_{0}\right), f_{\alpha}\left(x, y_{0}\right)<f\left(x, y_{0}\right)+\epsilon / 4<f\left(x, y^{\prime}\right)+\epsilon / 2<f_{\alpha}\left(x, y^{\prime}\right)+$ $3 \epsilon / 4$. Since $y_{\alpha} \rightarrow y_{0}$, there exists $\alpha_{1} \geq \alpha_{0}$ such that $y_{\alpha_{1}} \in O\left(y_{0}\right)$; it follows that $f\left(x, y_{0}\right)=f\left(x, y_{0}\right)-f_{\alpha_{1}}\left(x, y_{0}\right)+f_{\alpha_{1}}\left(x, y_{0}\right)-f_{\alpha_{1}}\left(x, y_{\alpha_{1}}\right)+f_{\alpha_{1}}\left(x, y_{\alpha_{1}}\right)<$ $\rho\left(f, f_{\alpha_{1}}\right)+3 \epsilon / 4<\epsilon$. Since $\epsilon>0$ is arbitrary, $f\left(x, y_{0}\right) \leq 0$ for all $x \in X$. This implies that $\left(f, y_{0}\right) \in \operatorname{Graph} F$ and hence Graph $F$ is closed in $M \times X$. Therefore $F$ is upper semicontinuous since $X$ is compact.

Definition 2.1. For each $f \in M$, (i) a point $y \in F(f)$ is $K F$-essential relative to $M$ if for each open neighborhood $N(y)$ of $y$ in $X$, there exists an open neighborhood $O(f)$ of $f$ in $M$ such that $F\left(f^{\prime}\right) \cap N(y) \neq \varnothing$ for each $f^{\prime} \in$ $O(f)$; (ii) $f$ is weakly essential relative to $M$ if there exists $y \in F(f)$ which is $K F$-essential relative to $M$; and (iii) $f$ is essential relative to $M$ if every $y \in F(f)$ is $K F$-essential relative to $M$.

The following result is due to Fort [5, Theorem 2]:

Lemma 2.3. If $X$ is (completely) metrizable, $Z$ is a Baire space, and $F: Z \rightarrow$ $K(X)$ is an usco mapping, then the set of points where $F$ is lower semicontinuous is a (dense) residual set in $Z$.

Theorem 2.1. (i) $F$ is almost lower semicontinuous at $f \in M$ if and only if $f$ is weakly essential relative to $M$.

(ii) $F$ is lower semicontinuous at $f \in M$ if and only if $f$ is essential relative to $M$.

(iii) $F$ is continuous at $f \in M$ if and only if $f$ is essential relative to $M$.

Proof. (i) $F$ is almost lower semicontinuous at $f \in M$ if and only if there exists $y \in F(f)$ such that $y$ is $K F$-essential relative to $M$ if and only if $f$ is weakly essential relative to $M$. 
(ii) $F$ is lower semicontinuous at $f \in M$ if and only if each $y \in F(f)$ is $K F$-essential relative to $M$ if and only if $f$ is essential relative to $M$.

(iii) This follows from (ii) and Lemma 2.2.

If $X$ is metrizable by a metric $d$, then the Vietoris topology on $K(X)$ coincides with the topology generated by the Hausdorff metric $h$ induced by $d$ (e.g., see [8, Corollary 4.2.3, p. 41]). Then $F$ is continuous at $f \in M$ if and only if for each $\epsilon>0$ there is $\delta>0$ such that for each $g \in M, h(F(f), F(g))<\epsilon$ whenever $\rho(f, g)<\delta$; i.e., $F(f)$ is stable: $F(g)$ is "close" to $F(f)$ whenever $g$ is "close" to $f$. Theorem 2.1 (iii) shows that $F(f)$ is stable if and only if $f$ is essential relative to $M$.

We shall give a sufficient condition that $f \in M$ is essential relative to $M$ :

Theorem 2.2. If $f \in M$ is such that $F(f)$ is a singleton set, then $f$ is essential relative to $M$.

Proof. Suppose $F(f)=\{x\}$. Let $G$ be any open set in $X$ such that $F(f) \cap$ $G \neq \varnothing$; then $x \in G$ so that $F(f) \subset G$. Since $F$ is upper semicontinuous at $f$ by Lemma 2.2, there is an open neighborhood $O(f)$ of $f$ in $M$ such that $F\left(f^{\prime}\right) \subset G$ for each $f^{\prime} \in O(f)$; in particular, $G \cap F\left(f^{\prime}\right) \neq \varnothing$ for each $f^{\prime} \in O(f)$. Thus $F$ is lower semicontinuous at $f$. By Theorem 2.1(ii), $f$ is essential relative to $M$.

Theorem 2.3. (i) Suppose that $X$ belongs to class $\mathscr{L}$. Then there exists a dense $G_{\delta}$ subset $Q$ of $M$ such that $f$ is weakly essential relative to $M$ for each $f \in Q$.

(ii) Suppose that $X$ is (completely) metrizable. Then there exists a (dense) residual subset $Q$ of $M$ such that $f$ is essential relative to $M$ for each $f \in Q$. Proof. (i) Since $M$ is a complete metric space, $M$ is Cech-complete. By Lemma 2.2, the mapping $F: M \rightarrow K(X)$ is upper semicontinuous. Since $X$ belongs to class $\mathscr{L}, F$ is almost lower semicontinuous on some (dense) $G_{\delta}$ subset $Q$ of $M$. By Theorem 2.1(i), $f$ is weakly essential relative to $M$ for each $f \in Q$.

(ii) By Lemma 2.2 and Lemma 2.3, $F$ is lower semicontinuous on some (dense) residual subset $Q$ of $M$. By Theorem 2.1(ii), $f$ is essential relative to $M$ for each $f \in Q$.

We remark that if we define $M=\{f \in L(X): f$ satisfies the conditions (i) and (ii) of Theorem $\mathrm{A}\}$ and $F(f)=\left\{y \in X: \sup _{x \in X} f(x, y) \leq\right.$ $\left.\sup _{x \in X} f(x, x)\right\}$ for each $f \in M$, then all results in this section remain valid.

\section{Stability in the NON-COMPACt SETting}

In section 2, we have studied the stability of the solution set $F(f)$ of $f$ in $X$ with $f$ varying but $X$ fixed. In this section we shall study the stability of the solution set $F(f) \cap A$ of $f$ in $A$ with both $f$ and $A$ varying.

Throughout this section, $X$ denotes a Hausdorff topological space and $L(X)$ denotes the space of all bounded real-valued lower semicontinuous functions on $X \times X$. For each $f_{1}, f_{2} \in L(X)$, let

$$
\rho\left(f_{1}, f_{2}\right)=\sup _{(x, y) \in X \times X}\left|f_{1}(x, y)-f_{2}(x, y)\right| ;
$$

then clearly $\rho$ is a metric on $L(X)$. 
Let $Y=K(X) \times L(X)$. Now for each $u=(A, f) \in Y$, we consider the following problem of finding $y \in A$ such that

$$
\sup _{x \in A} f(x, y) \leq 0 \text {. }
$$

Such a point $y$ in $A$ satisfying $\left({ }^{*}\right)$ is called a Ky Fan's point (in short, $K F$ point) of $f$ in $A$.

Before we study the stability of the set $F(u)$ of $K F$ points of $f$ in $A$ for $u=(A, f) \in Y$, we shall give several lemmas which will be used to prove our main results later. The proof of the following result is routine and is hence omitted.

Lemma 3.1. $(L(X), \rho)$ is a complete metric space.

Lemma 3.2. Suppose $X$ is a non-empty subset of a Hausdorff topological vector space. If $\left\{A_{\alpha}\right\}_{\alpha \in \Gamma}$ is a net of compact and convex sets in $K(X)$ which converges to $A \in K(X)$ in the Vietoris topology, then $A$ is also convex.

Proof. Suppose that $A$ were not convex. Then there exist $x_{1}, x_{2} \in A$ and $\lambda \in(0,1)$ such that $\lambda x_{1}+(1-\lambda) x_{2} \notin A$. Since $A$ is compact, there exist an open set $G$ in $X$ containing $A$ and an open neighborhood $O\left(\lambda x_{1}+(1-\lambda) x_{2}\right)$ of $\lambda x_{1}+(1-\lambda) x_{2}$ in $X$ such that $O\left(\lambda x_{1}+(1-\lambda) x_{2}\right) \cap G=\varnothing$. Note that there exist an open neighborhood $O\left(x_{1}\right)$ of $x_{1}$ in $X$ and an open neighborhood $O\left(x_{2}\right)$ of $x_{2}$ in $X$ such that $\lambda O\left(x_{1}\right)+(1-\lambda) O\left(x_{2}\right) \subset O\left(\lambda x_{1}+(1-\lambda) x_{2}\right)$. Since $x_{1}, x_{2} \in A$ and $A_{\alpha} \rightarrow A$, there exists $\alpha_{0} \in \Gamma$ such that for any $\alpha \geq \alpha_{0}$, $O\left(x_{1}\right) \cap A_{\alpha} \neq \varnothing$ and $O\left(x_{2}\right) \cap A_{\alpha} \neq \varnothing$. Since $G \supset A$, there exists $\alpha_{1} \in \Gamma$ such that for each $\alpha \geq \alpha_{1}, G \supset A_{\alpha}$. Now let $\alpha_{2} \in \Gamma$ be such that $\alpha_{2} \geq \alpha_{0}$ and $\alpha_{2} \geq \alpha_{1}$. Then for any $\alpha \geq \alpha_{2}, O\left(x_{1}\right) \cap A_{\alpha} \neq \varnothing, O\left(x_{2}\right) \cap A_{\alpha} \neq \varnothing$, and $A_{\alpha} \subset G$. Choose any $z_{1} \in O\left(x_{1}\right) \cap A_{\alpha_{2}}$ and $z_{2} \in O\left(x_{2}\right) \cap A_{\alpha_{2}}$. Since $A_{\alpha_{2}}$ is convex, $\lambda z_{1}+(1-\lambda) z_{2} \in A_{\alpha_{2}} \subset G$. But

$$
\lambda z_{1}+(1-\lambda) z_{2} \in \lambda O\left(x_{1}\right)+(1-\lambda) O\left(x_{2}\right) \subset O\left(\lambda x_{1}+(1-\lambda) x_{2}\right)
$$

which contradicts $O\left(\lambda x_{1}+(1-\lambda) x_{2}\right) \cap G=\varnothing$. Hence $A$ must be convex.

The following result is Lemma 3.3 of Beer [1]; as it was stated without a proof, we shall include its simple proof for completeness:

Lemma 3.3. Let $\left\{A_{\alpha}\right\}_{\alpha \in \Gamma}$ be a net in $K(X)$ which converges to $A \in K(X)$ in the Vietoris topology. Then any net $\left\{x_{\alpha}\right\}_{\alpha \in \Gamma}$ with $x_{\alpha} \in A_{\alpha}$ for each $\alpha \in \Gamma$ has a cluster point in $A$.

Proof. Suppose that the net $\left\{x_{\alpha}\right\}_{\alpha \in \Gamma}$ has no cluster point in $A$. Then for each $x \in A$, there exist an open neighborhood $O(x)$ of $x$ in $X$ and an $\alpha(x) \in \Gamma$ such that $x_{\alpha} \notin O(x)$ for all $\alpha \geq \alpha(x)$. Since $A \subset \cup_{x \in A} O(x)$ and $A$ is compact, there exist $x_{1}, x_{2}, \ldots, x_{n} \in A$ such that $A \subset \cup_{i=1}^{n} O\left(x_{i}\right)$. Now let $\alpha^{\prime}$ be such that $\alpha^{\prime} \geq \alpha\left(x_{i}\right)$ for $i=1,2, \ldots, n$. Then for any $\alpha \geq \alpha^{\prime}, x_{\alpha} \notin O\left(x_{i}\right)$ for $i=1,2, \ldots, n$. Since $\cup_{i=1}^{n} O\left(x_{i}\right)$ is an open set which contains $A$ and $A_{\alpha} \rightarrow A$ in the Vietoris topology, there exists $\alpha^{\prime \prime} \in \Gamma$ such that for any $\alpha \geq \alpha^{\prime \prime}, x_{\alpha} \in A_{\alpha} \subset \cup_{i=1}^{n} O\left(x_{i}\right)$. Now let $\alpha^{\prime \prime \prime} \in \Gamma$ be such that $\alpha^{\prime \prime \prime} \geq \alpha^{\prime}$ and $\alpha^{\prime \prime \prime} \geq \alpha^{\prime \prime}$; then $x_{\alpha^{\prime \prime \prime}} \notin O\left(x_{i}\right)$ for $i=1,2, \ldots, n$ which contradicts $x_{\alpha^{\prime \prime \prime}} \in A_{\alpha^{\prime \prime \prime}} \subset \cup_{i=1}^{n} O\left(x_{i}\right)$. Hence $\left\{x_{\alpha}\right\}_{\alpha \in \Gamma}$ has a cluster point in $A$. 
Now define the subspace $M$ of $Y$ by $M=\{(A, f) \in Y$ : there exists $y \in A$ such that $\left.\sup _{x \in A} f(x, y) \leq 0\right\}$. Then we define a mapping $F: M \rightarrow \mathscr{P}_{0}(X)$ by $F(u)=\left\{y \in A: \sup _{x \in A} f(x, y) \leq 0\right\}$ for each $u=(A, f) \in M$.

Lemma 3.4. $M$ is closed in $Y$.

Proof. Suppose that $\left\{\left(A_{\alpha}, f_{\alpha}\right)\right\}_{\alpha \in \Gamma}$ is a net in $M$ such that $\left(A_{\alpha}, f_{\alpha}\right) \rightarrow(A, f)$ $\in Y$. For each $\alpha \in \Gamma$, let $y_{\alpha} \in A_{\alpha}$ be such that $\sup _{x \in A_{\alpha}} f_{\alpha}\left(x, y_{\alpha}\right) \leq 0$. Since $A_{\alpha} \rightarrow A$ in the Vietoris topology, the net $\left\{y_{\alpha}\right\}_{\alpha \in \Gamma}$ has a cluster point $y_{0} \in A$ by Lemma 3.3. Now we shall show that $\sup _{x \in A} f\left(x, y_{0}\right) \leq 0$. Suppose that this is not true; then there would exist $\epsilon_{0}>0$ and $x_{0} \in A$ such that $f\left(x_{0}, y_{0}\right)>\varepsilon_{0}$. Since $f$ is lower semicontinuous at $\left(x_{0}, y_{0}\right)$, there exist an open neighborhood $O\left(x_{0}\right)$ of $x_{0}$ in $X$ and an open neighborhood $O\left(y_{0}\right)$ of $y_{0}$ in $X$ such that $f(x, y)>\epsilon_{0}$ for any $(x, y) \in O\left(x_{0}\right) \times O\left(y_{0}\right)$. Since $f_{\alpha} \rightarrow f$, there exists $\alpha_{0} \in \Gamma$ such that for any $\alpha \geq \alpha_{0},\left|f_{\alpha}(x, y)-f(x, y)\right|<\epsilon_{0} / 2$ for all $(x, y) \in X \times X$. Therefore $f_{\alpha}(x, y)>f(x, y)-\epsilon_{0} / 2>\epsilon_{0}-\epsilon_{0} / 2=\epsilon_{0} / 2$ for each $(x, y) \in O\left(x_{0}\right) \times O\left(y_{0}\right)$. As $A_{\alpha} \rightarrow A$, there exists $\alpha_{1} \geq \alpha_{0}$ such that $O\left(x_{0}\right) \cap A_{\alpha} \neq \varnothing$ for all $\alpha \geq \alpha_{1}$. Note that $y_{0} \in A$ is a cluster point of $\left\{y_{\alpha}\right\}_{\alpha \in \Gamma}$ and that there exists $\alpha_{2} \geq \alpha_{1}$ such that $y_{\alpha_{2}} \in O\left(y_{0}\right)$. Choose any $x_{\alpha_{2}} \in O\left(x_{0}\right) \cap A_{\alpha_{2}}$; we have $f_{\alpha_{2}}\left(x_{\alpha_{2}}, y_{\alpha_{2}}\right)>\varepsilon_{0} / 2$ which contradicts the choice of $y_{\alpha_{2}} \in A_{\alpha_{2}}$ that $\sup _{x \in A_{\alpha_{2}}} f_{\alpha}\left(x, y_{\alpha}\right) \leq 0$. Therefore we must have that $\sup _{x \in A} f\left(x, y_{0}\right) \leq 0$. Hence $(A, f) \in M$ and $M$ is thus closed in $Y$.

Lemma 3.5. If $X$ is Cech-complete, then $M$ is Cech-complete.

Proof. The space $L(X)$ is Cech-complete since $L(X)$ is a complete metric space by Lemma 3.1. Since $X$ is Cech-complete, $K(X)$ is also Cech-complete by Lemma 2.2 of Beer [1]. Therefore the product space $K(X) \times L(X)$ is Cechcomplete by Theorem 3.9.8 of Engelking [3]. By Lemma 3.4 and Theorem 3.9.6 of Engelking [3], $M$ is also Cech-complete.

Lemma 3.6. $F(u) \in K(X)$ for each $u \in M$.

Proof. For each $u=(A, f) \in M$, since $F(u) \subset A$, it is sufficient to prove that $F(u)$ is closed in $A$. Let $\left\{y_{\alpha}\right\}_{\alpha \in \Gamma}$ be a net in $F(u)$ which converges to a point $y_{0} \in A$. By the definition of $F$, we have $\sup _{x \in A} f\left(x, y_{\alpha}\right) \leq 0$ for each $\alpha \in \Gamma$. By the lower semicontinuity of $y \mapsto \sup _{x \in A} f(x, y)$, we have $\sup _{x \in A} f\left(x, y_{0}\right) \leq 0$. Hence $y_{0} \in F(u)$ so that $F(u)$ is a closed subset of $A$.

Lemma 3.7. The correspondence $F: M \rightarrow K(X)$ is upper semicontinuous.

Proof. Suppose that $F$ is not upper semicontinuous at some point $u=(A, f) \in$ $M$; then there would exist an open subset $G$ of $X$ with $G \supset F(u)$ and a net $\left\{u_{\alpha}\right\}_{\alpha \in \Gamma}$ in $M$ with $u_{\alpha} \rightarrow u \in M$ such that for each $\alpha \in \Gamma$, there exists $y_{\alpha} \in F\left(u_{\alpha}\right)$ with $y_{\alpha} \notin G$. Denote $u_{\alpha}=\left(A_{\alpha}, f_{\alpha}\right)$; then $f_{\alpha} \rightarrow f$ and $A_{\alpha} \rightarrow$ $A$. Since $y_{\alpha} \in A_{\alpha}$ for each $\alpha \in \Gamma$, by Lemma 3.3, the net $\left\{y_{\alpha}\right\}_{\alpha \in \Gamma}$ has a cluster point $y_{0} \in A$. Since $y_{\alpha} \notin G$ for each $\alpha \in \Gamma$, we have $y_{0} \notin G$. Therefore $\sup _{x \in A} f\left(x, y_{0}\right)>0$, so that there exist $\epsilon_{0}>0$ and $x_{0} \in A$ such that $f\left(x_{0}, y_{0}\right)>\epsilon_{0}$. Since $(x, y) \mapsto f(x, y)$ is lower semicontinuous at $\left(x_{0}, y_{0}\right)$, there exist an open neighborhood $O\left(x_{0}\right)$ of $x_{0}$ in $X$ and an open neighborhood $O\left(y_{0}\right)$ of $y_{0}$ in $X$ such that for any $(x, y) \in O\left(x_{0}\right) \times O\left(y_{0}\right), f(x, y)>\epsilon_{0}$. Since $f_{\alpha} \rightarrow f$, there exists $\alpha_{1} \in \Gamma$ such that for any $\alpha \geq \alpha_{1}, \mid f_{\alpha}(x, y)-$ $f(x, y) \mid<\epsilon_{0} / 2$ for any $(x, y) \in X \times X$. Therefore $f_{\alpha}(x, y)>f(x, y)-\epsilon_{0} / 2$ 
for all $(x, y) \in X \times X$. Since $O\left(x_{0}\right) \cap A \neq \varnothing$ and $A_{\alpha} \rightarrow A$, there exists $\alpha_{2} \geq \alpha_{1}$ such that for any $\alpha \geq \alpha_{2}, O\left(x_{0}\right) \cap A_{\alpha} \neq \varnothing$. Note that $y_{0}$ is a cluster point of the net $\left\{y_{\alpha}\right\}_{\alpha \in \Gamma}$ and that there exists $\alpha_{3} \geq \alpha_{2}$ with $y_{\alpha_{3}} \in O\left(y_{0}\right)$. Now choose any $x_{\alpha_{3}} \in O\left(x_{0}\right) \cap A_{\alpha_{3}}$; we have $f_{\alpha_{3}}\left(x_{\alpha_{3}}, y_{\alpha_{3}}\right)>f\left(x_{\alpha_{3}}, y_{\alpha_{3}}\right)-\epsilon_{0} / 2$. Therefore $f_{\alpha_{3}}\left(x_{\alpha_{3}}, y_{\alpha_{3}}\right)>f\left(x_{\alpha_{3}}, y_{\alpha_{3}}\right)-\epsilon_{0} / 2>\epsilon_{0}-\epsilon_{0} / 2=\epsilon_{0} / 2>0$ which contradicts the fact that $y_{\alpha_{3}} \in F\left(u_{\alpha_{3}}\right)$. Therefore $F$ must be upper semicontinuous.

Now let $M_{1}$ be a non-empty closed subset of $M$.

Definition 3.1. For each $u \in M_{1}$, (i) a point $y \in F(u)$ is $K F$-essential relative to $M_{1}$ if for each open neighborhood $O(y)$ of $y$ in $X$, there exists an open neighborhood $O(u)$ of $u$ in $M_{1}$ such that $F\left(u^{\prime}\right) \cap O(y) \neq \varnothing$ for each $u^{\prime} \in$ $O(u)$; (ii) $u$ is weakly essential relative to $M_{1}$ if there exists $y \in F(u)$ which is $K F$-essential relative to $M_{1}$; and (iii) $u$ is essential relative to $M_{1}$ if every $y \in F(u)$ is $K F$-essential relative to $M_{1}$.

Theorem 3.1. (i) $F$ is almost lower semicontinuous at $u \in M_{1}$ if and only if $u$ is weakly essential relative to $M_{1}$.

(ii) $F$ is lower semicontinuous at $u \in M_{1}$ if and only if $u$ is essential relative to $M_{1}$.

(iii) $F$ is continuous at $u \in M_{1}$ if and only if $u$ is essential relative to $M_{1}$.

Proof. (i) $F$ is almost lower semicontinuous at $u \in M_{1}$ if and only if there exists $y \in F(u)$ such that $y$ is $K F$-essential relative to $M_{1}$ if and only if $u$ is weakly essential relative to $M_{1}$.

(ii) $F$ is lower semicontinuous at $u \in M_{1}$ if and only if each $y \in F(u)$ is $K F$-essential relative to $M_{1}$ if and only if $u$ is essential relative to $M_{1}$.

(iii) This follows from (ii) and Lemma 3.7.

A proof analogous to that of Theorem 2.2 gives us the following result and is thus omitted:

Theorem 3.2. If $u \in M_{1}$ is such that $F(u)$ is a singleton set, then $u$ is essential relative to $M_{1}$.

Theorem 3.3. (i) Let $X$ be Cech-complete and belong to the class $\mathscr{L}$. Then there exists a dense $G_{\delta}$ subset $Q$ of $M_{1}$ such that $u$ is weakly essential relative to $M_{1}$ for each $u \in Q$.

(ii) Let $X$ be (completely) metrizable. Then there exists a (dense) residual subset $Q$ of $M_{1}$ such that $u$ is essential relative to $M_{1}$ for each $u \in Q$.

Proof. Note that $F$ is an usco by Lemma 3.6 and Lemma 3.7.

(i) Since $X$ is Cech-complete, Lemma 3.5 implies that $M$ is also Cechcomplete. Since $M_{1}$ is closed in $M, M_{1}$ is also Cech-complete by Theorem 3.9.6 [3]. Since $X \in \mathscr{L}$, there is a dense $G_{\delta}$ subset $Q$ of $M_{1}$ such that $F$ is almost lower semicontinuous at each $u \in Q$. By Theorem 3.1(i), $u$ is weakly essential relative to $M_{1}$ for each $u \in Q$.

(ii) By Lemma 2.3, there exists a (dense) residual subset $Q$ of $M_{1}$ such that $F$ is lower semicontinuous at each $u \in Q$. By Theorem 3.1(ii), $u$ is essential relative to $M_{1}$ for each $u \in Q$.

If $X$ is a complete metric space with metric $d$, then $K(X)$ is a complete metric space when equipped with the Hausdorff metric $h$ induced by $d$. By Corollary $4.2 .3[8$, p. 41$]$, the Vietoris topology on $K(X)$ coincides with the 
topology induced by the Hausdorff metric $h$. By Lemma 3.1, it follows that $Y=K(X) \times L(X)$ and hence $M$ and $M_{1}$ are also complete metric spaces when equipped with the metric $D$ defined by

$$
D\left(u, u^{\prime}\right)=\rho\left(f, f^{\prime}\right)+h\left(A, A^{\prime}\right)
$$

for $u=(A, f)$ and $u^{\prime}=\left(A^{\prime}, f^{\prime}\right)$. We note then, the mapping $F: M_{1} \rightarrow K(X)$ is continuous at $u=(A, f) \in M_{1}$ if and only if for each $\epsilon>0$, there is $\delta>0$ such that $h\left(F(u), F\left(u^{\prime}\right)\right)<\epsilon$ whenever $u^{\prime} \in M_{1}$ and $D\left(u, u^{\prime}\right)<\delta$; i.e., the solution set $F(u)$ of $u$ is stable: $F\left(u^{\prime}\right)$ is close to $F(u)$ whenever $u^{\prime}$ is close to $u$ for all $u^{\prime} \in M_{1}$. Theorem 3.1(iii) implies that if $u \in M_{1}$, then $u$ is essential relative to $M_{1}$ if and only if the solution set $F(u)$ is stable.

Now let $X$ be a non-empty closed and convex subset of a Frechet space $E$ equipped with a translation invariant metric $d$. Denote

$$
\begin{aligned}
& C K(X)=\{A \in K(X): A \text { is convex }\}, \\
& C L(X)=\{f \in L(X): f \text { satisfies (i) and (iii) of Theorem B }\} \\
& M^{\prime}=C K(X) \times C L(X) .
\end{aligned}
$$

The following is an application of the results obtained in this section:

Theorem 3.4. (i) $M^{\prime}$ is a non-empty closed subset of $M$.

(ii) There exists a dense $G_{\delta}$ subset $Q$ of $M^{\prime}$ such that $u$ is essential relative to $M^{\prime}$ for each $u \in Q$.

Proof. (i) Clearly $M^{\prime}$ is non-empty. If $u=(A, f) \in M^{\prime}$, then by Ky Fan's minimax inequality Theorem $\mathrm{B}$, there exists $\hat{y} \in A$ such that $\sup _{x \in A} f(x, \hat{y}) \leq$ 0 ; thus $u \in M$ so that $M^{\prime} \subset M$. Now if $\left\{\left(A_{n}, f_{n}\right)\right\}_{n=1}^{\infty}$ is a sequence in $M^{\prime}$ such that $\left(A_{n}, f_{n}\right) \rightarrow(A, f) \in M$, then $f_{n} \rightarrow f$ on $X$. Since for each $y \in X$, $x \mapsto f_{n}(x, y)$ is quasi-concave, it is also easy to see that $x \mapsto f(x, y)$ is also quasi-concave. By Lemma 3.2, $A$ is also convex. Thus $(A, f) \in M^{\prime}$ so that $M^{\prime}$ is closed in $M$.

Now (ii) follows from (i) and Theorem 3.3(ii).

Finally, we remark that if we define $M=\{(A, f) \in Y$ : there exists $y \in A$ such that $\left.\sup _{x \in A} f(x, y) \leq \sup _{x \in X} f(x, x)\right\}, F(u)=\left\{y \in A: \sup _{x \in A} f(x, y)\right.$ $\left.\leq \sup _{x \in X} f(x, x)\right\}$ for each $u=(A, f) \in M$ and $C L(X)=\{f \in L(X): f$ satisfies (i) and (ii) of Theorem A $\}$, then all results in this section remain valid.

For the study of stability of coincident points of upper semicontinuous multivalued mappings, stability of production economies, and the solutions in multiobjective optimization problems, we refer to the references [10], [11], and [12].

\section{REFERENCES}

1. G. Beer, On a generic optimization theorem of Kenderov, Nonlinear Anal. 12 (1988), 647-655.

2. J. P. Christensen, Theorems of Namioka and Johnson type for upper semicontinuous and compact valued set-valued mappings, Proc. Amer. Math. Soc. 86 (1982), 649-655.

3. R. Engelking, General topology, Polish Scientific, Warsaw, 1977.

4. K. Fan, A minimax inequality and its applications, Inequality III (O. Shisha, ed.), Academic Press, New York, 1972.

5. M. K. Fort, Jr., Points of continuity of semicontinuous functions, Publ. Math. Debrecen 2 (1951), 100-102. 
6. __ Essential and nonessential fixed points, Amer. J. Math. 72 (1950), 315-322.

7. P. S. Kenderov, Most of the optimization problems have unique solutions, Proceedings, Oberwolhfach Conference on Parametric Optimization (B. Brosowki and F. Deutsch, eds.), vol. 72, Birkhäuser, Basel, 1984, pp. 203-216.

8. E. Klein and A. Thompson, Theory of correspondences, Wiley, New York, 1984.

9. B. Lin and S. Simons, Nonlinear and convex analysis, Dekker, New York, 1987.

10. K.-K. Tan, J. Yu, and X.-Z. Yuan, The stability of coincident points for multivalued mappings, Nonlinear Anal. (to appear).

11. _ The stability of production economies, J. Austral. Math. Soc. (to appear).

12. J. Yu, Essential weak efficient solution in multiobjective optimization problems, J. Math. Anal. Appl. 166 (1992), 230-235.

(K.-K. Tan and X.-Z. Yuan) Department of Mathematics, Statistics and Computing Science, Dalhousie University, Halifax, Nova Scotia, Canada B3H 3J5

Current address, X.-Z. Yuan: Department of Mathematics, University of Queensland, Brisbane, Queensland 4072, Australia

E-mail address, K.-K. Tan: kktan৫cs.dal.ca

E-mail address, X.-Z. Yuan: xzy@axiom.maths.uq.oz.au

(J. Yu) Institute of Applied Mathematics, Guizhou Institute of Technology, Guiyang, Guizhou, CHINA 550003 